\title{
Optimal inequalities for a Toader-type mean by quadratic and contraharmonic means
}

\author{
Zhengchao Ji ${ }^{\mathrm{a}}$, Qing Ding ${ }^{\mathrm{b}, *}$, Tiehong Zhao ${ }^{\mathrm{c}}$ \\ ${ }^{a}$ Center of Mathematical Sciences, Zhejiang University, Hangzhou 310027, China. \\ ${ }^{b}$ College of Mathematics and Statistics, Hunan University of Finance and Economics, Changsha 410205, China. \\ ${ }^{c}$ Department of Mathematics, Hangzhou Normal University, Hangzhou 311121, China.
}

Communicated by M. Bohner

\begin{abstract}
In this paper, we present the best possible parameters $\alpha_{i}, \beta_{i}(i=1,2,3)$ and $\alpha_{4}, \beta_{4} \in(1 / 2,1)$ such that the double inequalities

$$
\begin{aligned}
& \alpha_{1} \mathrm{Q}(\mathrm{a}, \mathrm{b})+\left(1-\alpha_{1}\right) \mathrm{C}(\mathrm{a}, \mathrm{b})<\mathrm{T}_{\mathrm{Q}, \mathrm{C}}(\mathrm{a}, \mathrm{b})<\beta_{1} \mathrm{Q}(\mathrm{a}, \mathrm{b})+\left(1-\beta_{1}\right) \mathrm{C}(\mathrm{a}, \mathrm{b}), \\
& \mathrm{Q}^{\alpha_{2}}(\mathrm{a}, \mathrm{b}) \mathrm{C}^{1-\alpha_{2}}(\mathrm{a}, \mathrm{b})<\mathrm{T}_{\mathrm{Q}, \mathrm{C}}(\mathrm{a}, \mathrm{b})<\mathrm{Q}^{\beta_{2}}(\mathrm{a}, \mathrm{b}) \mathrm{C}^{1-\beta_{2}}(\mathrm{a}, \mathrm{b}), \\
& \frac{\mathrm{Q}(\mathrm{a}, \mathrm{b}) \mathrm{C}(\mathrm{a}, \mathrm{b})}{\alpha_{3} \mathrm{Q}(\mathrm{a}, \mathrm{b})+\left(1-\alpha_{3}\right) \mathrm{C}(\mathrm{a}, \mathrm{b})}<\mathrm{T}_{\mathrm{Q}, \mathrm{C}}(\mathrm{a}, \mathrm{b})<\frac{\mathrm{Q}(\mathrm{a}, \mathrm{b}) \mathrm{C}(\mathrm{a}, \mathrm{b})}{\beta_{3} \mathrm{Q}(\mathrm{a}, \mathrm{b})+\left(1-\beta_{3}\right) \mathrm{C}(\mathrm{a}, \mathrm{b})}, \\
& C\left(\sqrt{\alpha_{4} a^{2}+\left(1-\alpha_{4}\right) b^{2}}, \sqrt{\left(1-\alpha_{4}\right) a^{2}+\alpha_{4} b^{2}}\right)<T_{Q, C}(a, b)<C\left(\sqrt{\beta_{4} a^{2}+\left(1-\beta_{4}\right) b^{2}}, \sqrt{\left(1-\beta_{4}\right) a^{2}+\beta_{4} b^{2}}\right)
\end{aligned}
$$

hold for all $a, b>0$ with $a \neq b$, where $Q(a, b), C(a, b)$, and $T(a, b)$ are the quadratic, contraharmonic, and Toader means, respectively, and $\mathrm{T}_{\mathrm{Q}, \mathrm{C}}(\mathrm{a}, \mathrm{b})=\mathrm{T}[\mathrm{Q}(\mathrm{a}, \mathrm{b}), \mathrm{C}(\mathrm{a}, \mathrm{b})]$. As consequences, we provide new bounds for the complete elliptic integral of the second kind.

Keywords: Toader mean, elliptic integral, quadratic mean, contraharmonic mean.

2010 MSC: 26E60, 33E05.

(C)2018 All rights reserved.

\section{Introduction}

For $p \in \mathbb{R}$ and $a, b>0$ with $a \neq b$, the pth power mean $M_{p}(a, b)[7,9,17,18,22,32,35,37,38]$, pth Lehmer mean $L_{p}(a, b)[27,34]$, harmonic mean $H(a, b)$, geometric mean $G(a, b)$, arithmetic mean $A(a, b)$, Toader mean $T(a, b)[10,14,16,28]$, centroidal mean $\bar{C}(a, b)[6,36]$, quadratic mean $Q(a, b)[19]$,

\footnotetext{
*Corresponding author

Email addresses: jizhengchao@zju.edu.cn (Zhengchao Ji), dingqing@hufe.edu.cn (Qing Ding), tiehong.zhao@hznu.edu.cn (Tiehong Zhao)
}

doi: $10.22436 /$ jnsa.011.01.11

Received: 2017-05-16 Revised: 2017-11-09 Accepted: 2017-11-25 
contraharmonic mean $C(a, b)[5,13]$ are, respectively, defined by

$$
\begin{aligned}
& M_{p}(a, b)=\left\{\begin{array}{ll}
{\left[\left(a^{p}+b^{p}\right) / 2\right]^{1 / p},} & p \neq 0, \\
\sqrt{a b}, & p=0,
\end{array}, \quad L_{p}(a, b)=\frac{a^{p+1}+b^{p+1}}{a^{p}+b^{p}}\right. \\
& H(a, b)=\frac{2 a b}{a+b}, \quad G(a, b)=\sqrt{a b}, \quad A(a, b)=\frac{a+b}{2}, \\
& \mathrm{~T}(\mathrm{a}, \mathrm{b})=\frac{2}{\pi} \int_{0}^{\pi / 2} \sqrt{\mathrm{a}^{2} \cos ^{2} \theta+\mathrm{b}^{2} \sin ^{2} \theta} \mathrm{d} \theta, \quad \overline{\mathrm{C}}(\mathrm{a}, \mathrm{b})=\frac{2\left(\mathrm{a}^{2}+\mathrm{ab}+\mathrm{b}^{2}\right)}{3(\mathrm{a}+\mathrm{b})}, \\
& \mathrm{Q}(\mathrm{a}, \mathrm{b})=\sqrt{\frac{\mathrm{a}^{2}+\mathrm{b}^{2}}{2}}, \quad \mathrm{C}(\mathrm{a}, \mathrm{b})=\frac{\mathrm{a}^{2}+\mathrm{b}^{2}}{\mathrm{a}+\mathrm{b}} .
\end{aligned}
$$

It is well-known that $M_{p}(a, b)$ and $L_{p}(a, b)$ are continuous and strictly increasing with respect to $p \in \mathbb{R}$ for fixed $a, b>0$ with $a \neq b$, the following inequality chain

$$
\begin{aligned}
\mathrm{H}(\mathrm{a}, \mathrm{b}) & =\mathrm{M}_{-1}(\mathrm{a}, \mathrm{b})=\mathrm{L}_{-1}(\mathrm{a}, \mathrm{b})<\mathrm{G}(\mathrm{a}, \mathrm{b})=\mathrm{M}_{0}(\mathrm{a}, \mathrm{b})=\mathrm{L}_{-1 / 2}(\mathrm{a}, \mathrm{b}) \\
& <\mathrm{A}(\mathrm{a}, \mathrm{b})=\mathrm{M}_{1}(\mathrm{a}, \mathrm{b})=\mathrm{L}_{0}(\mathrm{a}, \mathrm{b})<\mathrm{T}(\mathrm{a}, \mathrm{b})<\overline{\mathrm{C}}(\mathrm{a}, \mathrm{b}) \\
& <\mathrm{Q}(\mathrm{a}, \mathrm{b})=\mathrm{M}_{2}(\mathrm{a}, \mathrm{b})<\mathrm{C}(\mathrm{a}, \mathrm{b})=\mathrm{L}_{1}(\mathrm{a}, \mathrm{b})
\end{aligned}
$$

holds for all $a, b>0$ with $a \neq b$.

The Toader mean $T(a, b)$ has been well known in the mathematical literature for many years (see $[20,21,24])$, which is related to the complete elliptic integral of the second kind $\varepsilon(r)=\int_{0}^{\pi / 2}(1-$ $\left.r^{2} \sin ^{2} \theta\right)^{1 / 2} \mathrm{~d} \theta(r \in(0,1))[12,15,25,30,31,33,39,40]$ and it can be rewritten as

$$
T(a, b)= \begin{cases}2 a \mathcal{E}\left(\sqrt{1-(b / a)^{2}}\right) / \pi, & a \geqslant b, \\ 2 b \mathcal{E}\left(\sqrt{1-(a / b)^{2}}\right) / \pi, & a<b .\end{cases}
$$

Let $r \in(0,1)$ and $r^{\prime}=\sqrt{1-r^{2}}$, then the complete elliptic integral of the first kind is given by $\mathcal{K}(r)=$ $\int_{0}^{\pi / 2}\left(1-r^{2} \sin ^{2} \theta\right)^{-1 / 2} d \theta$. We clearly see that $\mathcal{K}(r)$ is strictly increasing from $(0,1)$ onto $(\pi / 2,+\infty)$ and $\mathcal{E}(r)$ is strictly decreasing from $(0,1)$ onto $(1, \pi / 2)$. Moreover, $\mathcal{K}(r)$ and $\mathcal{E}(r)$ satisfy the following Landen identities and derivatives formulas (see [2, Appendix E, p.474-475])

$$
\begin{array}{ll}
\mathcal{K}\left(\frac{2 \sqrt{r}}{1+r}\right)=(1+r) \mathcal{K}(r), & \mathcal{E}\left(\frac{2 \sqrt{r}}{1+r}\right)=\frac{2 \mathcal{E}(r)-r^{\prime 2} \mathcal{K}(r)}{1+r}, \\
\frac{d \mathcal{K}(r)}{d r}=\frac{\mathcal{E}(r)-r^{\prime 2} \mathcal{K}(r)}{r r^{\prime 2}}, \quad \frac{d \mathcal{E}(r)}{d r}=\frac{\mathcal{E}(r)-\mathcal{K}(r)}{r}, \\
\frac{d\left[\mathcal{E}(r)-r^{\prime 2} \mathcal{K}(r)\right]}{d r}=r \mathcal{K}(r), \quad \frac{d[\mathcal{K}(r)-\mathcal{E}(r)]}{d r}=\frac{r \mathcal{E}(r)}{r^{\prime 2}}
\end{array}
$$

The special values $\mathcal{K}(\sqrt{2} / 2)$ and $\mathcal{E}(\sqrt{2} / 2)$ will be used later, which can be expressed as (see $[4$, Theorem 1.7])

$$
\mathcal{K}(\sqrt{2} / 2)=\frac{\Gamma^{2}(1 / 4)}{4 \sqrt{\pi}}=1.854 \cdots, \quad \mathcal{E}(\sqrt{2} / 2)=\frac{4 \Gamma^{2}(3 / 4)+\Gamma^{2}(1 / 4)}{8 \sqrt{\pi}}=1.350 \cdots,
$$

where $\Gamma(x)=\int_{0}^{\infty} t^{x-1} e^{-t} d t$ is the classical Euler's gamma function.

The special bivariate mean $T_{X, Y}(a, b)$ derived from Toader mean for any bivariate means $X(a, b)$ and $Y(a, b)$ of positive numbers $a, b$ is given by

$$
T_{X, Y}(a, b)=T[X(a, b), Y(a, b)]
$$

which is called a Toader-type mean. We denote the pairs of means $\{X, Y\}$ the generating means of the Toader-type mean defined in (1.4). 
Recently, the Toader mean has been the subject of intensive research. Vuorinen [29] conjectured that the inequality

$$
\mathrm{T}(\mathrm{a}, \mathrm{b})>\mathrm{M}_{3 / 2}(\mathrm{a}, \mathrm{b})
$$

holds for all $a, b>0$ with $a \neq b$. This conjecture was proved by Qiu and Shen [26], and Barnard et al. [3], respectively.

Alzer and Qiu [1] presented a best possible upper power mean bound of the Toader mean as follows

$$
\mathrm{T}(\mathrm{a}, \mathrm{b})<\mathrm{M}_{\log 2 /(\log \pi-\log 2)}(\mathrm{a}, \mathrm{b})
$$

for all $a, b>0$ with $a \neq b$.

Chu and Wang [11] prove the double inequality

$$
\mathrm{L}_{p}(\mathrm{a}, \mathrm{b})<\mathrm{T}(\mathrm{a}, \mathrm{b})<\mathrm{L}_{\mathrm{q}}(\mathrm{a}, \mathrm{b})
$$

holds for all $a, b>0$ with $a \neq b$ if and only $p \leqslant 0$ and $q \geqslant 1 / 4$.

Very recently, optimal bounds for $T_{A, C}(a, b)$ and $T_{A, Q}(a, b)$ by several convex combinations of their generating means were established. Li et al. [23] presented the best possible parameters $\alpha_{i}$ and $\beta_{i}$ with $i=1,2,3,4$ such that the double inequalities

$$
\begin{aligned}
& \alpha_{1} A(a, b)+\left(1-\alpha_{1}\right) C(a, b)<T_{A, C}(a, b)<\beta_{1} A(a, b)+\left(1-\beta_{1}\right) C(a, b), \\
& A^{\alpha_{2}}(a, b) C^{1-\alpha_{2}}(a, b)<T_{A, C}(a, b)<A^{\beta_{2}}(a, b) C^{1-\beta_{2}}(a, b), \\
& \frac{\alpha_{3}}{A(a, b)}+\frac{1-\alpha_{3}}{C(a, b)}<\frac{1}{T_{A, C}(a, b)}<\frac{\beta_{3}}{A(a, b)}+\frac{1-\beta_{3}}{C(a, b)}, \\
& C\left(\alpha_{4} a+\left(1-\alpha_{4}\right) b, \alpha_{4} b+\left(1-\alpha_{4}\right) a\right)<T_{A, C}(a, b)<C\left(\beta_{4} a+\left(1-\beta_{4}\right) b, \beta_{4} b+\left(1-\beta_{4}\right) a\right)
\end{aligned}
$$

hold for all $a, b>0$ with $a \neq b$.

In [8], the authors found the best possible parameters $\lambda_{1}, \mu_{1}, \lambda_{2}, \mu_{2} \in(1 / 2,1)$ such that the double inequalities

$$
\begin{aligned}
& \mathrm{Q}\left(\mathrm{a}, \mathrm{b} ; \lambda_{1}\right)<\mathrm{T}_{\mathrm{A}, \mathrm{Q}}(\mathrm{a}, \mathrm{b})<\mathrm{Q}\left(\mathrm{a}, \mathrm{b} ; \mu_{1}\right), \\
& \mathrm{C}\left(\mathrm{a}, \mathrm{b} ; \lambda_{2}\right)<\mathrm{T}_{\mathrm{A}, \mathrm{Q}}(\mathrm{a}, \mathrm{b})<\mathrm{C}\left(\mathrm{a}, \mathrm{b} ; \mu_{2}\right)
\end{aligned}
$$

hold for all $a, b>0$ with $a \neq b$, where $M(a, b ; p)=M[p a+(1-p) b, p b+(1-p) a]$ is the one-parameter mean of $a$ and $b$. Besides, another expression of optimal bounds for $T_{A, Q}(a, b)$ was given by Zhao et al. [41]. Explicitly, they proved the double inequality

$$
\left[\alpha(r) A^{r}(a, b)+(1-\alpha(r)) Q^{r}(a, b)\right]^{1 / r}<T_{A, Q}(a, b)<\left[\beta(r) A^{r}(a, b)+(1-\beta(r)) Q^{r}(a, b)\right]^{1 / r}
$$

holds for all $r \leqslant 1$ and $a, b>0$ with $a \neq b$ if and only if $\alpha(r) \geqslant 1 / 2$ and $\beta(r) \leqslant \lambda(r)$, where $\lambda(r)$ is defined by $\lambda(r)=\left[1-(2 \mathcal{E}(\sqrt{2} / 2) / \pi)^{r}\right] /\left[1-(\sqrt{2} / 2)^{r}\right](r \neq 0)$ and $\lambda(0)=\log [2 \varepsilon(\sqrt{2} / 2) / \pi] / \log (\sqrt{2} / 2)$.

From (1.1) and (1.2) together with the properties of a mean, we clearly see that

$$
\mathrm{Q}(\mathrm{a}, \mathrm{b})<\mathrm{T}_{\mathrm{Q}, \mathrm{C}}(\mathrm{a}, \mathrm{b})<\mathrm{C}(\mathrm{a}, \mathrm{b}) \text {. }
$$

for all $a, b>0$ with $a \neq b$.

Motivated by inequality (1.5) and the results of [8], it is natural to ask what are the best possible parameters $\alpha_{i}, \beta_{i}(i=1,2,3)$ and $\alpha_{4}, \beta_{4} \in(1 / 2,1)$ such that the double inequalities

$$
\begin{aligned}
\alpha_{1} \mathrm{Q}(\mathrm{a}, \mathrm{b})+\left(1-\alpha_{1}\right) \mathrm{C}(\mathrm{a}, \mathrm{b}) & <\mathrm{T}_{\mathrm{Q}, \mathrm{C}}(\mathrm{a}, \mathrm{b})<\beta_{1} \mathrm{Q}(\mathrm{a}, \mathrm{b})+\left(1-\beta_{1}\right) \mathrm{C}(\mathrm{a}, \mathrm{b}), \\
\mathrm{Q}^{\alpha_{2}}(\mathrm{a}, \mathrm{b}) \mathrm{C}^{1-\alpha_{2}}(\mathrm{a}, \mathrm{b}) & <\mathrm{T}_{\mathrm{Q}, \mathrm{C}}(\mathrm{a}, \mathrm{b})<\mathrm{Q}^{\beta_{2}}(\mathrm{a}, \mathrm{b}) \mathrm{C}^{1-\beta_{2}}(\mathrm{a}, \mathrm{b}), \\
\mathrm{Q}(\mathrm{a}, \mathrm{b}) \mathrm{C}(\mathrm{a}, \mathrm{b}) & <\mathrm{T}_{\mathrm{Q}, \mathrm{C}}(\mathrm{a}, \mathrm{b})<\frac{\mathrm{Q}(\mathrm{a}, \mathrm{b}) \mathrm{C}(\mathrm{a}, \mathrm{b})}{\beta_{3} \mathrm{Q}(\mathrm{a}, \mathrm{b})+\left(1-\beta_{3}\right) \mathrm{C}(\mathrm{a}, \mathrm{b})}, \\
\mathrm{C}\left(\sqrt{\alpha_{4} \mathrm{a}^{2}+\left(1-\alpha_{4}\right) \mathrm{b}^{2}}, \sqrt{\left(1-\alpha_{4}\right) \mathrm{a}^{2}+\alpha_{4} \mathrm{~b}^{2}}\right) & <\mathrm{T}_{\mathrm{Q}, \mathrm{C}}(\mathrm{a}, \mathrm{b})<\mathrm{C}\left(\sqrt{\beta_{4} \mathrm{a}^{2}+\left(1-\beta_{4}\right) \mathrm{b}^{2}}, \sqrt{\left(1-\beta_{4}\right) \mathrm{a}^{2}+\beta_{4} \mathrm{~b}^{2}}\right)
\end{aligned}
$$

hold for all $a, b>0$ with $a \neq b$ ? The main purpose of this paper is to answer this question. 


\section{Lemmas}

In order to prove the desired theorem, we need several lemmas which we present in this section.

Lemma 2.1 ([2, Theorem 1.25]). For $-\infty<\mathrm{a}<\mathrm{b}<\infty$, let $\mathrm{f}, \mathrm{g}:[\mathrm{a}, \mathrm{b}] \rightarrow \mathbb{R}$ be continuous on $[\mathrm{a}, \mathrm{b}]$, and be differentiable on $(\mathrm{a}, \mathrm{b})$, let $\mathrm{g}^{\prime}(\mathrm{x}) \neq 0$ on $(\mathrm{a}, \mathrm{b})$. If $\mathrm{f}^{\prime}(\mathrm{x}) / \mathrm{g}^{\prime}(\mathrm{x})$ is increasing (decreasing) on $(\mathrm{a}, \mathrm{b})$, then so are

$$
\frac{f(x)-f(a)}{g(x)-g(a)} \text { and } \frac{f(x)-f(b)}{g(x)-g(b)} \text {. }
$$

If $\mathrm{f}^{\prime}(\mathrm{x}) / \mathrm{g}^{\prime}(\mathrm{x})$ is strictly monotone, then the monotonicity in the conclusion is also strict.

\section{Lemma 2.2.}

(1) $[\mathcal{K}(\mathrm{r})-\mathcal{E}(\mathrm{r})] / \mathrm{r}^{2}$ is strictly increasing on $(0,1)$;

(2) $[\mathcal{K}(r)-\mathcal{E}(r)] / \log \left(1 / r^{\prime}\right)$ is strictly decreasing from $(0,1)$ onto $(1, \pi / 2)$;

(3) $r^{\prime c} \mathcal{E}(r)$ is strictly increasing on $(0,1)$ if and only if $c \leqslant-1 / 2$.

Proof. Parts (1)-(3) follow from [2, Exercise 3.43 (11) and (15), Theorem 3.21 (8)].

Lemma 2.3. Let $\delta_{1}=2[\pi-2 \varepsilon(\sqrt{2} / 2)] /(2-\sqrt{2}) \pi=0.4785 \cdots$ and

$$
f(r)=\frac{1-2 \mathcal{E}(r) / \pi}{1-\sqrt{1-r^{2}}}
$$

then $f(r)$ is strictly decreasing from $(0, \sqrt{2} / 2)$ onto $\left(\delta_{1}, 1 / 2\right)$.

Proof. Let $f_{1}(r)=1-2 \mathcal{E}(r) / \pi$ and $f_{2}(r)=1-\sqrt{1-r^{2}}$. Then we clearly see that $f_{1}(0)=f_{2}(0)=0$ and $f(r)=f_{1}(r) / f_{2}(r)$.

Taking the derivative of $f_{1}(r)$ and $f_{2}(r)$ yields

$$
\frac{f_{1}^{\prime}(r)}{f_{2}^{\prime}(r)}=\frac{2}{\pi} \cdot \frac{\mathcal{K}(r)-\mathcal{E}(r)}{\log \left(1 / r^{\prime}\right)} \cdot \frac{r^{\prime} \log \left(1 / r^{\prime}\right)}{1-r^{\prime 2}}
$$

An easy calculation yields

$$
\frac{\mathrm{d}\left[\mathrm{r}^{\prime} \log \left(1 / \mathrm{r}^{\prime}\right) /\left(1-\mathrm{r}^{\prime 2}\right)\right]}{\mathrm{d} \mathrm{r}^{\prime}}=\frac{\left(1-\mathrm{r}^{\prime 2}\right)+\left(1+\mathrm{r}^{\prime 2}\right) \log \mathrm{r}^{\prime}}{\left(1-\mathrm{r}^{\prime 2}\right)^{2}}>0
$$

for $r^{\prime} \in(0,1)$.

It follows from (2.2) together with the monotonicity of $r^{\prime}=\sqrt{1-r^{2}}$ that $r^{\prime} \log \left(1 / r^{\prime}\right) /\left(1-r^{\prime 2}\right)$ is strictly decreasing on $(0,1)$ with respect to $r$. This conjunction with $(2.1)$ and Lemma $2.2(2)$ implies that $f_{1}^{\prime}(r) / f_{2}^{\prime}(r)$ is strictly decreasing on $(0,1)$. Therefore, Lemma 2.3 follows from Lemma 2.1 and the limiting values $f\left(0^{+}\right)=1 / 2$ and $f\left(\frac{\sqrt{2}}{2}^{-}\right)=\delta_{1}$.

Lemma 2.4. Let $\delta_{2}=2 \log [\pi /(2 \mathcal{E}(\sqrt{2} / 2))] / \log 2=0.435698 \cdots$ and

$$
g(r)=\frac{2[\log (2 / \pi)+\log \mathcal{E}(r)]}{\log \left(1-r^{2}\right)}
$$

then $\mathrm{g}(\mathrm{r})$ is strictly decreasing from $(0, \sqrt{2} / 2)$ onto $\left(\delta_{2}, 1 / 2\right)$.

Proof. Let $g_{1}(r)=2[\log (2 / \pi)+\log \mathcal{E}(r)]$ and $g_{2}(r)=\log \left(1-r^{2}\right)$, then it is easy to see that $g_{1}(0)=g_{2}(0)=$ $0, g(r)=g_{1}(r) / g_{2}(r)$ and

$$
\frac{g_{1}^{\prime}(r)}{g_{2}^{\prime}(r)}=\frac{g_{11}(r)}{g_{22}(r)}
$$

where $g_{11}(r)=\left(1-r^{2}\right)[\mathcal{K}(r)-\varepsilon(r)]$ and $g_{22}(r)=r^{2} \varepsilon(r)$. 
Observe that $g_{11}(0)=g_{22}(0)=0$. Taking the derivative of $g_{11}(r)$ and $g_{22}(r)$ yields

$$
\frac{g_{11}^{\prime}(r)}{g_{22}^{\prime}(r)}=\frac{3 \mathcal{E}(r)-2 \mathcal{K}(r)}{3 \mathcal{E}(r)-\mathcal{K}(r)}=1-\frac{1}{3 \mathcal{E}(r) / \mathcal{K}(r)-1} .
$$

It follows from (2.4) and the monotonicity of $\mathcal{E}(r) / \mathcal{K}(r)$ that $g_{11}^{\prime}(r) / g_{22}^{\prime}(r)$ is strictly decreasing on $(0,1)$. This conjunction with (2.3) and Lemma 2.1 implies that $g(r)$ is strictly decreasing on $(0,1)$.

The proof of Lemma 2.4 is completed from the limit values $g\left(0^{+}\right)=1 / 2$ and $g\left(\frac{\sqrt{2}}{2}^{-}\right)=\delta_{2}$.

Lemma 2.5. Let $\delta_{3}=[\sqrt{2} \mathcal{E}(\sqrt{2} / 2)-\pi / 2] /(\sqrt{2}-1) \mathcal{E}(\sqrt{2} / 2)=0.606488 \cdots$ and

$$
\mathrm{h}(\mathrm{r})=\frac{\pi /[2 \mathcal{E}(\mathrm{r})]-1 / \sqrt{1-\mathrm{r}^{2}}}{1-1 / \sqrt{1-\mathrm{r}^{2}}}
$$

then $\mathrm{h}(\mathrm{r})$ is strictly increasing from $(0, \sqrt{2} / 2)$ onto $\left(1 / 2, \delta_{3}\right)$.

Proof. Let $h_{1}(r)=\pi /[2 \mathcal{E}(r)]-1 / \sqrt{1-r^{2}}$ and $h_{2}(r)=1-1 / \sqrt{1-r^{2}}$, then we clearly see that $h_{1}(0)=$ $h_{2}(0)=0, h(r)=h_{1}(r) / h_{2}(r)$ and

$$
\frac{h_{1}^{\prime}(r)}{h_{2}^{\prime}(r)}=1-\frac{\pi}{2} \cdot \frac{r^{\prime}[\mathcal{K}(r)-\mathcal{E}(r)]}{1-r^{\prime 2}} \cdot \frac{1}{\left[r^{\prime-1} \mathcal{E}(r)\right]^{2}}
$$

Lemma 2.2 (3) and (2.1) together with the proof of Lemma 2.3 lead to the conclusion that $1 /\left[r^{\prime-1} \mathcal{E}(r)\right]^{2}$ and $r^{\prime}[\mathcal{K}(r)-\mathcal{E}(r)] /\left(1-r^{\prime 2}\right)$ are strictly decreasing on $(0,1)$. This conjunction with (2.5) implies that $h_{1}^{\prime}(r) / h_{2}^{\prime}(r)$ is strictly increasing on $(0,1)$.

Therefore, Lemma 2.5 follows immediately from Lemma 2.1 and the limiting values $h\left(0^{+}\right)=1 / 2$ and $h\left(\frac{\sqrt{2}}{2}^{-}\right)=\delta_{3}$.

Lemma 2.6. The function $\varphi(r)=\left[1-r^{2} / 4-2 \mathcal{E}(r) / \pi\right] / r^{4}$ is strictly increasing from $(0, \sqrt{2} / 2)$ onto $(3 / 64,7 / 2-$ $8 \mathcal{E}(\sqrt{2} / 2) / \pi)$.

Proof. Let $\varphi_{1}(r)=1-r^{2} / 4-2 \varepsilon(r) / \pi$ and $\varphi_{2}(r)=r^{4}$, then $\varphi_{1}(0)=\varphi_{2}(0)=0, \varphi(r)=\varphi_{1}(r) / \varphi_{2}(r)$, and

$$
\frac{\varphi_{1}^{\prime}(r)}{\varphi_{2}^{\prime}(r)}=\frac{\mathcal{K}(r)-\mathcal{E}(r)-\pi / 4}{2 \pi r^{4}}
$$

Taking the derivate of (2.6) yields

$$
\frac{\mathrm{d}\left[\varphi_{1}^{\prime}(\mathrm{r}) / \varphi_{2}^{\prime}(\mathrm{r})\right]}{\mathrm{dr}}=\frac{\mu(\mathrm{r})}{2 \pi r^{5}\left(1-\mathrm{r}^{2}\right)^{\prime}}
$$

where

$$
\mu(r)=\left(4-3 r^{2}\right) \mathcal{E}(r)+\left(1-r^{2}\right)[\pi-4 \mathcal{K}(r)] .
$$

An easy calculation leads to

$$
\mu(\sqrt{2} / 2)=1.23926 \cdots, \quad \mu^{\prime}(r)=r[7 \mathcal{K}(r)-9 \mathcal{E}(r)-2 \pi]<r[7 \mathcal{K}(\sqrt{2} / 2)-9 \mathcal{E}(\sqrt{2} / 2)-2 \pi]<-5 r
$$

for $r \in(0, \sqrt{2} / 2)$. It follows from (2.8) that $\mu(r)>0$ for $r \in(0, \sqrt{2} / 2)$. This conjunction with (2.7) implies that $\varphi_{1}^{\prime}(\mathrm{r}) / \varphi_{2}^{\prime}(\mathrm{r})$ is strictly increasing on $(0, \sqrt{2} / 2)$.

Therefore, Lemma 2.6 follows from Lemma 2.1 together with the limit values $\varphi\left(0^{+}\right)=3 / 64$ and $\varphi\left(\frac{\sqrt{2}}{2}^{-}\right)=7 / 2-8 \mathcal{E}(\sqrt{2} / 2) / \pi$.

Notice that $7 / 2-8 \mathcal{E}(\sqrt{2} / 2) / \pi=0.0606 \cdots<1 / 8$, then from Lemma 2.6 we can get the following corollary directly. 
Corollary 2.7. The double inequality

$$
1-\frac{r^{2}}{4}-\frac{r^{4}}{8}<\frac{2}{\pi} \varepsilon(r)<1-\frac{r^{2}}{4}-\frac{3 r^{4}}{64}
$$

holds for $r \in(0, \sqrt{2} / 2)$.

Lemma 2.8. Let $\delta_{4}=\left[\pi \sqrt{2 \mathcal{E}^{2}(\sqrt{2} / 2)-\pi^{2} / 4}\right] / \mathcal{E}^{2}(\sqrt{2} / 2)=1.87157 \cdots, \lambda \in(0,2]$ and

$$
\Phi_{\lambda}(r)=\frac{\sqrt{1+\lambda r r^{\prime}}-\sqrt{1-\lambda r r^{\prime}}}{\lambda r}
$$

then the following statements are true:

(1) $\Phi_{\sqrt{2}}(r)<1-r^{2} / 4-r^{4} / 4$ holds for $r \in(0, \sqrt{2} / 2)$;

(2) $\Phi_{\delta_{4}}(r)>1-r^{2} / 4$ holds for $r \in(0,17 / 25)$;

(3) $2 \varepsilon(r) / \pi+r / 2$ is strictly increasing on $(0, \sqrt{2} / 2)$;

(4) $\Phi_{\delta_{4}}(r)+r / 2$ is strictly decreasing on $(17 / 25, \sqrt{2} / 2)$.

Proof.

(1). We first claim that

$$
\sqrt{1-2 r^{2}+2 r^{4}}>1-r^{2}+\frac{r^{4}}{2}+\frac{r^{6}}{2}
$$

for $r \in(0, \sqrt{2} / 2)$. Indeed, it follows easily from

$$
1-2 r^{2}+2 r^{4}-\left(1-r^{2}+\frac{r^{4}}{2}+\frac{r^{6}}{2}\right)^{2}=\frac{r^{8}}{4}\left(1-r^{2}\right)\left(r^{2}+3\right)>0
$$

for $r \in(0, \sqrt{2} / 2)$. From (2.9), we clearly see that

$$
\begin{aligned}
\Phi_{\sqrt{2}}^{2}(r)-\left(1-\frac{r^{2}}{4}-\frac{r^{4}}{4}\right)^{2} & =\frac{1-\sqrt{1-2 r^{2}+2 r^{4}}}{r^{2}}-\left(1-\frac{r^{2}}{4}-\frac{r^{4}}{4}\right)^{2} \\
& <1-\frac{r^{2}}{2}-\frac{r^{4}}{2}-\left(1-\frac{r^{2}}{4}-\frac{r^{4}}{4}\right)^{2}=-\frac{r^{4}\left(r^{2}+1\right)}{16}<0 .
\end{aligned}
$$

This completes the proof of Lemma 2.8 (1).

(2). In order to prove that $\Phi_{\delta_{4}}(r)>1-r^{2} / 4$ for $r \in(0,17 / 25)$, by squaring both sides of the inequality and simplifying, it suffices to show that

$$
\sqrt{1-\delta_{4}^{2} r^{2}\left(1-r^{2}\right)}<1-\frac{\delta_{4}^{2}}{2} r^{2}\left(1-\frac{r^{2}}{4}\right)^{2}
$$

holds for $r \in(0,17 / 25)$.

We consider the difference of both sides squares of (2.10) as follows

$$
1-\delta_{4}^{2} r^{2}\left(1-r^{2}\right)-\left[1-\frac{\delta_{4}^{2}}{2} r^{2}\left(1-\frac{r^{2}}{4}\right)^{2}\right]^{2}=-\frac{\delta_{4}^{2} r^{4}}{1024} \eta(r),
$$

where

$$
\eta(r)=\delta_{4}^{2} r^{8}-16 \delta_{4}^{2} r^{6}+96 \delta_{4}^{2} r^{4}-256 \delta_{4}^{2} r^{2}-64 \delta_{4}^{2} r^{2}+256 \delta_{4}^{2}-512
$$


An easy calculation yields

$$
\eta(17 / 25)=6.9982 \cdots, \quad \eta^{\prime}(r)=-8 r\left[\delta_{4}^{2}\left(4-r^{2}\right)^{3}+16\right]<0 .
$$

It follows from (2.12) that $\eta(r)>0$ for $r \in(0,17 / 25)$. This conjunction with (2.11) completes the proof of Lemma 2.8 (2).

(3). It suffices to determine the sign of the derivate of $2 \mathcal{E}(r) / \pi+r / 2$. An easy computation yields

$$
\frac{\mathrm{d}[2 \mathcal{E}(\mathrm{r}) / \pi+\mathrm{r} / 2]}{\mathrm{d} r}=\frac{1}{2}-\frac{2 \mathrm{r}}{\pi} \cdot \frac{\mathcal{K}(\mathrm{r})-\mathcal{E}(\mathrm{r})}{\mathrm{r}^{2}} .
$$

It follows from (2.13) and Lemma 2.2 (1) that $d[2 \mathcal{E}(r) / \pi+r / 2] / d r$ is strictly decreasing on $(0,1)$. The monotonicity of (2.13) leads to the conclusion that

$$
\frac{\mathrm{d}[2 \mathcal{E}(\mathrm{r}) / \pi+\mathrm{r} / 2]}{\mathrm{dr}}>\frac{1}{2}-\frac{2 \sqrt{2}}{\pi}[\mathcal{K}(\sqrt{2} / 2)-\mathcal{E}(\sqrt{2} / 2)]=0.046753 \cdots
$$

This completes the proof.

(4). Let $\omega_{1}(r)=\sqrt{1+\delta_{4} r r^{\prime}}$ and $\omega_{2}(r)=\sqrt{1-\delta_{4} r r^{\prime}}$, then $\omega_{1}(r)>\omega_{2}(r)>0$ for $r \in(0, \sqrt{2} / 2)$. By easy computations, one has

$$
\begin{aligned}
\frac{d\left[\omega_{1}(r)-\omega_{2}(r)\right]}{d r} & =\frac{\delta_{4}\left(1-2 r^{2}\right)}{2 \sqrt{1-r^{2}}}\left[\frac{1}{\omega_{1}(r)}+\frac{1}{\omega_{2}(r)}\right]>0, \\
\frac{d\left[1 / \omega_{1}(r)+1 / \omega_{2}(r)\right]}{d r} & =\frac{\delta_{4}\left(1-2 r^{2}\right)}{2 \sqrt{1-r^{2}}}\left[\frac{1}{\omega_{2}^{3}(r)}-\frac{1}{\omega_{1}^{3}(r)}\right]>0
\end{aligned}
$$

for $r \in(0, \sqrt{2} / 2)$.

Moreover, it is easy to see that $\left(1-2 r^{2}\right) / \sqrt{1-r^{2}}=2 \sqrt{1-r^{2}}-1 / \sqrt{1-r^{2}}$ is strictly decreasing on $(0, \sqrt{2} / 2)$. This conjunction with (2.14) implies that

$$
\begin{aligned}
\frac{d\left[\Phi_{\delta_{4}}(r)+r / 2\right]}{d r}=- & \frac{\omega_{1}(r)-\omega_{2}(r)}{\delta_{4} r^{2}}+\frac{1-2 r^{2}}{2 \sqrt{1-r^{2}}}\left[\frac{1}{\omega_{1}(r)}+\frac{1}{\omega_{2}(r)}\right]+\frac{1}{2} \\
<- & \frac{\omega_{1}(17 / 25)-\omega_{2}(17 / 25)}{\delta_{4} r^{2}} \\
& +\frac{1-2 \times(17 / 25)^{2}}{2 \sqrt{1-(17 / 25)^{2}}}\left[\frac{1}{\omega_{1}(\sqrt{2} / 2)}+\frac{1}{\omega_{2}(\sqrt{2} / 2)}\right]+\frac{1}{2} \\
< & -\frac{3}{5 r^{2}}+\frac{1}{4 r}+\frac{1}{2} \\
< & \frac{1}{r^{2}}\left[-\frac{3}{5}+\frac{1}{4} \times \frac{\sqrt{2}}{2}+\frac{1}{2} \times\left(\frac{\sqrt{2}}{2}\right)^{2}\right]=-\frac{14-5 \sqrt{2}}{40 r^{2}}<0
\end{aligned}
$$

for $r \in(17 / 25, \sqrt{2} / 2)$. Therefore, Lemma 2.8 (4) follows directly from (2.15).

\section{Main results}

Theorem 3.1. The double inequality

$$
\alpha_{1} \mathrm{Q}(\mathrm{a}, \mathrm{b})+\left(1-\alpha_{1}\right) \mathrm{C}(\mathrm{a}, \mathrm{b})<\mathrm{T}_{\mathrm{Q}, \mathrm{C}}(\mathrm{a}, \mathrm{b})<\beta_{1} \mathrm{Q}(\mathrm{a}, \mathrm{b})+\left(1-\beta_{1}\right) \mathrm{C}(\mathrm{a}, \mathrm{b})
$$

holds for all $\mathrm{a}, \mathrm{b}>0$ with $\mathrm{a} \neq \mathrm{b}$ if and only if $\alpha_{1} \geqslant 1 / 2$ and $\beta_{1} \leqslant \delta_{1}=2[\pi-2 \varepsilon(\sqrt{2} / 2)] /(2-\sqrt{2}) \pi=0.4785 \cdots$. 
Proof. Since $\mathrm{Q}(\mathrm{a}, \mathrm{b}), \mathrm{C}(\mathrm{a}, \mathrm{b})$, and $\mathrm{T}(\mathrm{a}, \mathrm{b})$ are symmetric and homogeneous of degree 1 , without loss of generality, we may assume that $a>b>0$. Let $r=(a-b) / \sqrt{2\left(a^{2}+b^{2}\right)} \in(0, \sqrt{2} / 2)$, then (1.1) and (1.3) lead to

$$
Q(a, b)=\frac{A(a, b)}{\sqrt{1-r^{2}}}, \quad C(a, b)=\frac{A(a, b)}{1-r^{2}}, \quad T_{Q, C}(a, b)=\frac{2 A(a, b)}{\pi\left(1-r^{2}\right)} \varepsilon(r)
$$

It follows from (3.1) that

$$
\frac{C(a, b)-T_{Q, C}(a, b)}{C(a, b)-Q(a, b)}=\frac{1-2 \mathcal{E}(r) / \pi}{1-\sqrt{1-r^{2}}}=f(r),
$$

where $f(r)$ is defined as in Lemma 2.3. Therefore, Theorem 3.1 follows easily from Lemma 2.3 and (3.2).

Theorem 3.2. The double inequality

$$
\mathrm{Q}^{\alpha_{2}}(\mathrm{a}, \mathrm{b}) \mathrm{C}^{1-\alpha_{2}}(\mathrm{a}, \mathrm{b})<\mathrm{T}_{\mathrm{Q}, \mathrm{C}}(\mathrm{a}, \mathrm{b})<\mathrm{Q}^{\beta_{2}}(\mathrm{a}, \mathrm{b}) \mathrm{C}^{1-\beta_{2}}(\mathrm{a}, \mathrm{b})
$$

holds for all $\mathrm{a}, \mathrm{b}>0$ with $\mathrm{a} \neq \mathrm{b}$ if and only if $\alpha_{2} \geqslant 1 / 2$ and $\beta_{2} \leqslant \delta_{2}=2 \log [\pi /(2 \varepsilon(\sqrt{2} / 2))] / \log 2=$ $0.435698 \cdots$.

Proof. Without loss of generality, we assume that $a>b>0$. Let $r=(a-b) / \sqrt{2\left(a^{2}+b^{2}\right)} \in(0, \sqrt{2} / 2)$, then from (3.1) we clearly see that

$$
\frac{\log C(a, b)-\log T_{Q, C}(a, b)}{\log C(a, b)-\log Q(a, b)}=g(r),
$$

where $g(r)$ is defined as in Lemma 2.4 .

Therefore, Theorem 3.2 follows directly from (3.3) and Lemma 2.4.

Theorem 3.3. The double inequality

$$
\frac{\mathrm{Q}(\mathrm{a}, \mathrm{b}) \mathrm{C}(\mathrm{a}, \mathrm{b})}{\alpha_{3} \mathrm{Q}(\mathrm{a}, \mathrm{b})+\left(1-\alpha_{3}\right) \mathrm{C}(\mathrm{a}, \mathrm{b})}<\mathrm{T}_{\mathrm{Q}, \mathrm{C}}(\mathrm{a}, \mathrm{b})<\frac{\mathrm{Q}(\mathrm{a}, \mathrm{b}) \mathrm{C}(\mathrm{a}, \mathrm{b})}{\beta_{3} \mathrm{Q}(\mathrm{a}, \mathrm{b})+\left(1-\beta_{3}\right) \mathrm{C}(\mathrm{a}, \mathrm{b})}
$$

holds for all $\mathrm{a}, \mathrm{b}>0$ with $\mathrm{a} \neq \mathrm{b}$ if and only if $\alpha_{3} \leqslant 1 / 2$ and $\beta_{3} \geqslant \delta_{3}=[\sqrt{2} \mathcal{E}(\sqrt{2} / 2)-\pi / 2] /(\sqrt{2}-1) \mathcal{E}(\sqrt{2} / 2)=$ $0.606488 \cdots$.

Proof. In order to prove the double inequality in Theorem 3.3, it suffices to find $\alpha_{3}$ and $\beta_{3}$ such that

$$
\alpha_{3}<\frac{\frac{1}{T_{Q}, c(a, b)}-\frac{1}{Q(a, b)}}{\frac{1}{C(a, b)}-\frac{1}{Q(a, b)}}<\beta_{3}
$$

holds for all $a, b>0$ with $a \neq b$.

Without loss of generality, we assume that $a>b>0$. Let $r=(a-b) / \sqrt{2\left(a^{2}+b^{2}\right)} \in(0, \sqrt{2} / 2)$, then (3.1) leads to

$$
\frac{\frac{1}{T_{Q, c}(a, b)}-\frac{1}{Q(a, b)}}{\frac{1}{C(a, b)}-\frac{1}{Q(a, b)}}=h(r),
$$

where $h(r)$ is defined as in Lemma 2.5. Therefore, Theorem 3.3 follows directly from (3.4), (3.5), and Lemma 2.5. 
Theorem 3.4. Let $\alpha_{4}, \beta_{4} \in(1 / 2,1)$, then the double inequality

$$
\begin{aligned}
C\left(\sqrt{\alpha_{4} a^{2}+\left(1-\alpha_{4}\right) b^{2}}, \sqrt{\left(1-\alpha_{4}\right) a^{2}+\alpha_{4} b^{2}}\right) & <T_{Q, C}(a, b) \\
& <C\left(\sqrt{\beta_{4} a^{2}+\left(1-\beta_{4}\right) b^{2}}, \sqrt{\left(1-\beta_{4}\right) a^{2}+\beta_{4} b^{2}}\right)
\end{aligned}
$$

holds for all $\mathrm{a}, \mathrm{b}>0$ with $\mathrm{a} \neq \mathrm{b}$ if and only if $\alpha_{4} \leqslant(\sqrt{2}+2) / 4$ and $\beta_{4} \geqslant\left(\delta_{4}+2\right) / 4=0.967894 \cdots$, where $\delta_{4}$ is defined as in Lemma 2.8.

Proof. Since $\mathrm{T}(\mathrm{a}, \mathrm{b})$ and $\mathrm{C}(\mathrm{a}, \mathrm{b})$ are symmetric and homogeneous of degree one, we assume that $\mathrm{a}>\mathrm{b}>0$. Let $r=(a-b) / \sqrt{2\left(a^{2}+b^{2}\right)} \in(0, \sqrt{2} / 2)$, then (1.1), (3.1) lead to

$$
\begin{aligned}
& T_{Q, C}(a, b)-C\left(\sqrt{p a^{2}+(1-p) b^{2}}, \sqrt{p b^{2}+(1-p) a^{2}}\right) \\
& =C(a, b)\left[\frac{2}{\pi} \mathcal{E}(r)-\frac{\sqrt{1+(4 p-2) r r^{\prime}}-\sqrt{1-(4 p-2) r r^{\prime}}}{(4 p-2) r}\right]=C(a, b)\left[\frac{2}{\pi} \mathcal{E}(r)-\Phi_{4 p-2}(r)\right],
\end{aligned}
$$

where $\Phi_{\lambda}(r)$ is defined as in Lemma 2.8 .

It is easy to be verified that $C\left(\sqrt{p a^{2}+(1-p) b^{2}}, \sqrt{p b^{2}+(1-p) a^{2}}\right)$ is continuous and strictly increasing on $[1 / 2,1]$ with respect to $p$ for fixed $a, b>0$ with $a \neq b$.

We divide the proof into three cases.

Case 1. $p_{1}=(\sqrt{2}+2) / 4$. Then it follows from 2.7 and Lemma 2.8 (1) that

$$
\frac{2}{\pi} \varepsilon(r)-\Phi_{4 p_{1}-2}(r)=\frac{2}{\pi} \varepsilon(r)-\Phi_{\sqrt{2}}(r)>1-\frac{r^{4}}{4}-\frac{r^{4}}{8}-\left(1-\frac{r^{4}}{4}-\frac{r^{4}}{4}\right)=\frac{r^{4}}{8}>0
$$

for $r \in(0, \sqrt{2} / 2)$. Therefore, $T_{Q, C}(a, b)>C\left(\sqrt{p_{1} a^{2}+\left(1-p_{1}\right) b^{2}}, \sqrt{\left(1-p_{1}\right) a^{2}+p_{1} b^{2}}\right)$ follows from (3.6) and (3.7).

Case 2. $p_{2}=\left(\delta_{4}+2\right) / 4$. Then from Corollary 2.7 and Lemma 2.8 (2) we clearly see that

$$
\frac{2}{\pi} \varepsilon(r)-\Phi_{4 p_{2}-2}(r)=\frac{2}{\pi} \varepsilon(r)-\Phi_{\delta_{4}}(r)<1-\frac{r^{4}}{4}-\frac{3 r^{4}}{64}-\left(1-\frac{r^{4}}{4}\right)=-\frac{3 r^{4}}{64}<0
$$

for $r \in(0,17 / 25)$. Furthermore, it follows from Lemma 2.8 (3) and (4) that $2 \mathcal{E}(r) / \pi-\Phi_{\delta_{4}}(r)=2 \mathcal{E}(r) / \pi+$ $r / 2-\left[\Phi_{\delta_{4}}(r)+r / 2\right]$ is strictly increasing on $(17 / 25, \sqrt{2} / 2)$. This implies

$$
\frac{2}{\pi} \varepsilon(r)-\Phi_{4 p_{2}-2}(r)<\frac{2}{\pi} \varepsilon(\sqrt{2} / 2)-\Phi_{4 p_{2}-2}(\sqrt{2} / 2)=0
$$

for $r \in(17 / 25, \sqrt{2} / 2)$.

Therefore, $T_{Q, C}(a, b)<C\left(\sqrt{p_{2} a^{2}+\left(1-p_{2}\right) b^{2}}, \sqrt{\left(1-p_{2}\right) a^{2}+p_{2} b^{2}}\right)$ follows from (3.6), (3.8), and (3.9).

Case 3. $(\sqrt{2}+2) / 4<p_{3}<\left(\delta_{4}+2\right) / 4$. On the one hand, if $r \rightarrow 0$, then making use of Taylor series yields

$$
\frac{2}{\pi} \mathcal{E}(\mathrm{r})-\Phi_{4 \mathrm{p}_{3}-2}(\mathrm{r})=-2\left[\left(\mathrm{p}_{3}-\frac{2-\sqrt{2}}{4}\right)\left(\mathrm{p}_{3}-\frac{\sqrt{2}+2}{4}\right)\right] \mathrm{r}^{2}+\mathrm{o}\left(\mathrm{r}^{4}\right) .
$$

Equations (3.6) and (3.10) lead to the conclusion that there exists small enough $\tau_{1} \in(0, \sqrt{2} / 2)$ such that $\mathrm{T}_{\mathrm{Q}, \mathrm{C}}(\mathrm{a}, \mathrm{b})<\mathrm{C}\left(\sqrt{\mathrm{p}_{3} \mathrm{a}^{2}+\left(1-\mathrm{p}_{3}\right) \mathrm{b}^{2}}, \sqrt{\left(1-\mathrm{p}_{3}\right) \mathrm{a}^{2}+\mathrm{p}_{3} \mathrm{~b}^{2}}\right)$ for all $\mathrm{a}>\mathrm{b}>0$ with $(\mathrm{a}-\mathrm{b}) / \sqrt{2\left(a^{2}+\mathrm{b}^{2}\right)} \in$ $\left(0, \tau_{1}\right)$. 
On the other hand, it is easy to see that

$$
\frac{2}{\pi} \mathcal{E}(\sqrt{2} / 2)-\Phi_{4 p_{3}-2}(\sqrt{2} / 2)=\frac{2}{\pi} \mathcal{E}(\sqrt{2} / 2)+\frac{\sqrt{1-p_{3}}-\sqrt{p_{3}}}{2 p_{3}-1}
$$

is strictly decreasing on $(1 / 2,1)$ with respect to $p_{3}$. This implies that

$$
\frac{2}{\pi} \varepsilon(\sqrt{2} / 2)-\Phi_{4 p_{3}-2}(\sqrt{2} / 2)<\frac{2}{\pi} \varepsilon(\sqrt{2} / 2)-\Phi_{\delta_{4}}(\sqrt{2} / 2)=0 .
$$

Equations (3.6) and (3.11) lead to the conclusion that there exists small enough $\tau_{2} \in(0, \sqrt{2} / 2)$ such that $\mathrm{T}_{\mathrm{Q}, \mathrm{C}}(\mathrm{a}, \mathrm{b})>\mathrm{C}\left(\sqrt{\mathrm{p}_{3} \mathrm{a}^{2}+\left(1-\mathrm{p}_{3}\right) \mathrm{b}^{2}}, \sqrt{\left(1-\mathrm{p}_{3}\right) \mathrm{a}^{2}+\mathrm{p}_{3} \mathrm{~b}^{2}}\right)$ for all $\mathrm{a}>\mathrm{b}>0$ with $(\mathrm{a}-\mathrm{b}) / \sqrt{2\left(a^{2}+\mathrm{b}^{2}\right)} \in$ $\left(\sqrt{2} / 2-\tau_{2}, \sqrt{2} / 2\right)$.

The following corollary follows from Theorems 3.1, 3.2, 3.3, and 3.4 immediately.

Corollary 3.5. Let $\mathrm{r}^{\prime}=\sqrt{1-\mathrm{r}^{2}}$ and

$$
\begin{aligned}
& m(r)=\max \left\{\frac{1+r^{\prime}}{2}, \sqrt{r^{\prime}}, \frac{2 r^{\prime}}{1+r^{\prime}}, \frac{\sqrt{1+\sqrt{2} r r^{\prime}}-\sqrt{1-\sqrt{2} r r^{\prime}}}{\sqrt{2} r}\right\}, \\
& M(r)=\min \left\{1-\delta_{1}+\delta_{1} r^{\prime}, r^{\prime} \delta_{2}, \frac{r^{\prime}}{1-\delta_{3}+\delta_{3} r^{\prime}}, \frac{\sqrt{1+\delta_{4} r r^{\prime}}-\sqrt{1-\delta_{4} r r^{\prime}}}{\delta_{4} r}\right\},
\end{aligned}
$$

where $\delta_{1}, \delta_{2}, \delta_{3}$, and $\delta_{4}$ are defined as in Lemmas $2.3,2.4,2.5,2.8$, respectively. Then the double inequality

$$
\frac{\pi}{2} \mathrm{~m}(\mathrm{r})<\mathcal{E}(\mathrm{r})<\frac{\pi}{2} M(r)
$$

holds for all $r \in(0, \sqrt{2} / 2)$.

\section{Acknowledgment}

This work was supported by the National Natural Science Foundation of China (Grant No. 11301127), the general project Foundation of the department of Education of Hunan province (Grant No. 16C0265) and teaching reform research project in Hunan University of Finance and Economics (2016xjjg001).

\section{References}

[1] H. Alzer, S.-L. Qiu, Monotonicity theorems and inequalities for the complete elliptic integrals, J. Comput. Appl. Math., 172 (2004), 289-312. 1

[2] G. D. Anderson, M. K. Vamanamurthy, M. K. Vuorinen, Conformal Invariants, Inequalities, and Quasiconformal Maps, John Wiley \& Sons, New York, (1997). 1, 2.1, 2

[3] R. W. Barnard, K. Pearce, K. C. Richards, An inequality involving the generalized hypergeometric function and the arc length of an ellipse, SIAM J. Math. Anal., 31 (2000), 693-699. 1

[4] J. M. Borwein, P. B. Borwein, Pi and the AGM, John Wiley \& Sons, NewYork, (1987). 1

[5] Y.-M. Chu, S.-W. Hou, Sharp bounds for Seiffert mean in terms of contraharmonic mean, Abstr. Appl. Anal., 2012 (2012), 6 pages. 1

[6] Y.-M. Chu, S. W. Hou, W.-F. Xia, Optimal convex combinations bounds of centroidal and harmonic means for logarithmic and identric means, Bull. Iranian Math. Soc., 39 (2013), 259-269. 1

[7] Y.-M. Chu, B.-Y. Long, Bounds of the Neuman-Sándor mean using power and identic means, Abstr. Appl. Anal., 2013 (2013), 6 pages. 1

[8] H.-H. Chu, W.-M. Qian, Y.-M. Chu, Y.-Q. Song, Optimal bounds for a Toader-type mean in terms of one-parameter quadratic and contraharmonic means, J. Nonlinear Sci. Appl., 9 (2016), 3424-3432. 1, 1

[9] Y.-M. Chu, Y.-F. Qiu, M.-K. Wang, Sharp power mean bounds for the combination of Seiffert and geometric means, Abstr. Appl. Anal., 2010 (2010), 12 pages. 1 
[10] Y.-M. Chu, M.-K. Wang, Inequalities between arithmetic-geometric, Gini, and Toader means, Abstr. Appl. Anal., 2012 (2012), 11 pages. 1

[11] Y.-M. Chu, M.-K. Wang, Optimal Lehmer mean bounds for the Toader mean, Results Math., 61 (2012), 223-229. 1

[12] Y.-M. Chu, M.-K. Wang, Y.-P. Jiang, S.-L. Qiu, Concavity of the complete elliptic integrals of the second kind with respect to Hölder means, J. Math. Anal. Appl., 395 (2012), 637-642. 1

[13] Y.-M. Chu, M.-K. Wang, X.-Y. Ma, Sharp bounds for Toader mean in terms of contraharmonic mean with applications, J. Math. Inequal., 7 (2013), 161-166. 1

[14] Y.-M. Chu, M.-K. Wang, S.-L. Qiu, Optimal combinations bounds of root-square and arithmetic means for Toader mean, Proc. Indian Acad. Sci. Math. Sci., 122 (2012), 41-51. 1

[15] Y.-M. Chu, M.-K. Wang, S.-L. Qiu, Y.-P. Jiang, Bounds for complete elliptic integrals of the second kind with applications, Comput. Math. Appl., 63 (2012), 1177-1184. 1

[16] Y.-M. Chu, M.-K. Wang, S.-L. Qiu, Y.-F. Qiu, Sharp generalized Seiffert mean bounds for Toader mean, Abstr. Appl. Anal., 2011 (2011), 8 pages. 1

[17] Y.-M. Chu, W.-F. Xia, Two sharp inequalities for power mean, geometric mean, and harmonic mean, J. Inequal. Appl., 2009 (2009), 6 pages. 1

[18] Y.-M. Chu, W.-F. Xia, Two optimal double inequalities between power mean and logarithmic mean, Comput. Math. Appl., 60 (2010), 83-89. 1

[19] Z.-J. Guo, Y. Zhang, Y.-M. Chu, Y.-Q. Song, Sharp bounds for Neuman means in terms of geometric, arithmetic and quadratic means, J. Math. Inequal., 10 (2016), 301-312. 1

[20] H. Kazi, E. Neuman, Inequalities and bounds for elliptic integrals, J. Approx. Theory, 146 (2007), 212-226. 1

[21] H. Kazi, E. Neuman, Inequalities and bounds for elliptic integrals II, Amer. Math. Soc., 471 (2008), 127-138. 1

[22] Y.-M. Li, B.-Y. Long, Y.-M. Chu, Sharp bounds by the power mean for the generalized Heronian mean, J. Inequal. Appl., 2012 (2012), 9 pages. 1

[23] N. Li, T. Zhao, Y. Zhao, Bounds for a Toader-type mean by arithmetic and contraharmonic means, Int. J. Pure Appl. Math., 105 (2015), 257-268. 1

[24] E. Neuman, Bounds for symmetric elliptic integrals, J. Approx. Theory, 122 (2003), 249-259. 1

[25] W.-M. Qian, Y.-M. Chu, Sharp bounds for a special quasi- arithmetic mean in terms of arithmetic and geometric means with two parameters, J. Inequal. Appl., 2017 (2017), 10 pages. 1

[26] S. L. Qiu, J. M. Shen, On two problems concerning means, J. Hangzhou Inst. Electron. Eng., 17 (1997), 1-7. 1

[27] Y.-F. Qiu, M.-K. Wang, Y.-M. Chu, G.-D. Wang, Two sharp inequalities for Lehmer mean, identric mean and logarithmic mean, J. Math. Inequal., 5 (2011), 301-306. 1

[28] G. H. Toader, Some mean values related to the arithmetic-geometric mean, J. Math. Anal. Appl., 218 (1998), 358-368. 1

[29] M. Vuorinen, Hypergeometric functions in geometric function theory, Special Functions and Differential Equations, 119-126, (1998). 1

[30] M.-K. Wang, Y.-M. Chu, Asymptotical bounds for complete elliptic integrals of the second kind, J. Math. Anal. Appl., 402 (2013), 119-126. 1

[31] M.-K. Wang, Y.-M. Chu, Refinenemts of transformation inequalities for zero-balanced hypergeometric functions, Acta Math. Sci., 37 (2017), 607-622. 1

[32] M.-K. Wang, Y.-M. Chu, Y.-F. Qiu, S.-L. Qiu, An optimal power mean inequality for the complete elliptic integrals, Appl. Math. Lett., 24 (2011), 887-890. 1

[33] M.-K. Wang, Y.-M. Li, Y.-M. Chu, Inequalities and infinite product formula for Ramanujan generalized modular equation function, Ramanujan J., 2017 (2017), 12 pages. 1

[34] M.-K. Wang, Y.-F. Qiu, Y.-M. Chu, Sharp bounds for Seiffert means in terms of Lehmer means, J. Math. Inequal., 4 (2010), 581-586. 1

[35] G.-D. Wang, X.-H. Zhang, Y.-M. Chu, A power mean inequality for the Grötzsch ring function, Math. Inequal. Appl., 14 (2011), 833-837. 1

[36] W.-F. Xia, Y.-M. Chu, Optimal inequalities between Neuman- Sándor, centroidal and harmonic means, J. Math. Inequal., 7 (2013), 593-600. 1

[37] W.-F. Xia, Y.-M. Chu, G.-D. Wang, The optimal upper and lower power mean bounds for a convex combination of the arithmetic and logarithmic means, Abstr. Appl. Anal., 2010 (2010), 9 pages. 1

[38] W.-F. Xia, W. Janous, Y.-M. Chu, The optimal convex combination bounds of arithmetic and harmonic means in terms of power mean, J. Math. Inequal., 6 (2012), 241-248. 1

[39] Z.-H. Yang, Y.-M. Chu, A monotonicity property involving the generalized elliptic integral of the first kind, Math. Inequal. Appl., 20 (2017), 729-735. 1

[40] Z.-H. Yang, W.-M. Qian, Y.-M. Chu, W. Zhang, Monotonicity rule for the quotient of two functions and its application, J. Inequal. Appl., 2017 (2017), 13 pages. 1

[41] T.-H. Zhao, Y.-M. Chu, W. Zhang, Optimal inequalities for bounding Toader mean by arithmetic and quadratic means, J. Inequal. Appl., 2017 (2017), 10 pages. 1 